Marta Lizandra do Rêgo LEAL $^{1}$

Pierre Castro SOARES ${ }^{2}$

Heloísa Godoi

BERTAGNON ${ }^{1}$

Paulo Eduardo Gomes da SILVA $^{1}$

Enrico Lippi ORTOLANI ${ }^{1}$

Fernando José BENESI ${ }^{1}$

\section{Correspondência para:}

MARTA LIZANDRA DO RÊGO LEAL

Av. prof. Dr. Orlando Marques de Paiva, 87 05508-000-São Paulo-SP.

Recebido para publicação: 05/11/2004 Aprovado para publicação: 10/12/2006

\title{
Efeito da refrigeração sobre o exame hemogasométrico em sangue venoso de ovinos
}

\author{
1 - Departamento de Clínica Médica da Faculdade de Medicina Veterinária e \\ Zootecnia da Universidade de São Paulo, São Paulo - SP \\ 2 - Universidade Federal Rural de Pernambuco, UFRPE
}

\section{Resumo}

Palavras-chave:

Ovinos.

Com o propósito de avaliar o efeito da refrigeração sobre o exame hemogasométrico, foram utilizados 12 ovinos machos, hígidos, da raça Santa Inês, com cerca de quatro meses de idade e peso variando entre 30 e $45 \mathrm{~kg}$. As amostras de sangue destinadas ao exame hemogasométrico foram coletadas em duplicata utilizando-se agulhas descartáveis acopladas à seringas plásticas contendo cerca de 1000UI de heparina sódica. Durante e após a coleta tomou-se o cuidado de evitar a entrada de bolhas de ar no interior da seringa. As amostras não conservadas foram mantidas a temperatura ambiente, entre $23 \mathrm{e}$ $25^{\circ} \mathrm{C}$, e aquelas destinadas à refrigeração foram acondicionadas em isopor contendo $3 \mathrm{~kg}$ de água gelada e $3 \mathrm{~kg}$ de gelo, mantendo-se assim uma temperatura entre 0 e $4^{\circ} \mathrm{C}$. As análises hemogasométricas foram determinadas imediatamente após coleta e com 1,2,3,4,5,6,8,10,12 e 24 horas. As análises dos resultados indicaram alterações significativas nas amostras mantidas a temperatura ambiente, caracterizado-se por diminuições, a partir da 4, 8 e 10 horas após coleta, para os valores do $\mathrm{pH}, \mathrm{BE}$ e StB, respectivamente, e por aumento, a partir da 6 hora, para os valores da $\mathrm{PCO}_{2}$. Com relação as amostras conservadas, não foram evidenciadas variações significativas dos parâmetros ao longo dos tempos de análise. Conclui-se, portanto, que amostras de sangue venoso de ovinos são viáveis, para a realização do exame hemogasométrico, até 24 horas após coleta, desde que mantidas sob adequada refrigeração.

\section{Introdução}

O exame hemogasométrico, o qual inclui a análise dos gases sangüíneos e de demais variáveis necessárias para a avaliação do equilíbrio ácido-básico, é de grande importância para o diagnóstico e tratamento de enfermidades que afetam diferentes espécies animais, particularmente os ruminantes ${ }^{1}$. Dentre as alterações do exame hemogasométrico é importante destacar a acidose metabólica, caracterizada por diminuições do $\mathrm{pH}$ e dos teores de bicarbonato, sendo essa alteração observada, principalmente, nos casos de acidose láctica ruminal, toxemia da prenhez, diarréias, insuficiência renal e respiratória ${ }^{2}$.

O tempo de transporte e acondicio- namento de amostras biológicas, destinadas a tal exame devem ser levados em consideração, pois influenciam sobremaneira nos resultados das análises. $\mathrm{Na}$ Medicina Veterinária, muitas vezes, a realização de exames hemogasométricos, imediatamente após a coleta do sangue, torna-se pouco prático, particularmente para o profissional que trabalha a campo., ${ }^{2,3}$

Resultados sobre análises hemogasométricas em amostras de sangue sob refrigeração foram reportados por alguns autores: em bovinos ${ }^{1,4,5,6,7}$; em ovinos ${ }^{7,8} ; \mathrm{em}$ eqüinos $^{7,9}$; em suínos ${ }^{3}$ e em caninos $^{7,10}$.

As conclusões destes trabalhos demonstraram haver diferenças de opiniões a respeito do intervalo de tempo em que as amostras poderiam ser mantidas quanto a 
sua viabilidade. As indicações chegam a variar de seis a 24 horas após a coleta, quando estas amostras foram mantidas a 0 $-4^{0} \mathrm{C}$.

$\mathrm{Na}$ espécie ovina, no que tange as publicações disponíveis na literatura internacional, apenas dois trabalhos fazem referência do efeito da refrigeração na análise de variáveis hemogasométrica ${ }^{7,8}$ enquanto que no Brasil não existe citação bibliográfica. Dada a carência dessas informações na literatura o presente trabalho objetivou avaliar o exame hemogasométrico em amostras de sangue venoso de ovinos, mantidas a temperatura ambiente $\left(23\right.$ a $25^{\circ}$ C) ou entre 0 a $4^{0} \mathrm{C}$, ao longo do período de 24 horas.

\section{Material e Métodos}

Para o presente estudo foram utilizados 12 ovinos machos, hígidos, da raça Santa Inês, com cerca de quatro meses de idade e peso variando entre 30 e $40 \mathrm{~kg}$. Esses animais foram alojados em gaiolas individuais instaladas no Galpão de Experimentação da Clínica de Ruminantes do Departamento de Clínica Médica da Faculdade de Medicina Veterinária e Zootecnia da Universidade de São Paulo e foram alimentados com $200 \mathrm{~g}$ de concentrado comercial, por dia, além de terem feno e água "ad libitum". Antes do início do experimento os ovinos foram mantidos em suas instalações por um período de 90 dias para a adaptação à dieta e ao local de permanência.

No dia em que antecedeu o experimento aferiu-se a temperatura retal de cada animal, bem como foram coletados cinco $\mathrm{ml}$ de sangue da veia jugular externa para a determinação da hemoglobina, utilizando-se sistema de coleta a vácuo, em tubos de vidros siliconizados (vacutainer ${ }^{\circledR}$ ) contendo o EDTA como anticoagulante.

As amostras de sangue destinadas ao exame hemogasométrico foram coletadas em duplicata, de cada animal, por venopunção jugular externa, utilizando-se agulhas descartáveis $25 \mathrm{~mm} \times 0,8 \mathrm{~mm}$ acopladas a seringas plásticas com capacidade para $10 \mathrm{ml}$ contendo cerca de 1000UI de heparina sódica (liquemineROCHE) Durante e após a coleta tomouse o cuidado de evitar a entrada de bolhas de ar no interior da seringa e, logo após a punção do sangue, vedou-se a ponta da agulha com uma rolha de borracha, a fim de evitar a entrada ou saída de gases.

Após coleta, as seringas foram transportadas imediatamente para o Laboratório de Pesquisa do Departamento de Clínica Médica da FMVZ-USP. As amostras não conservadas foram mantidas à temperatura ambiente, entre 23 e $25^{\circ} \mathrm{C}$, e aquelas destinadas a refrigeração foram acondicionadas em isopor contendo 3 litros de água gelada e $3 \mathrm{Kg}$ de gelo reciclável, mantendo-se, assim, uma temperatura entre 0 e $3^{\circ} \mathrm{C}$. Com o intuito de evitar que a temperatura da água excede-se os $3^{\circ} \mathrm{C}$ um termômetro foi colocado em contato com a água gelada durante todo o período de estudo.

A dosagem da hemoglobina, efetuada um dia antes do experimento, foi realizada pelo método da cianohemoglobina, utilizando-se reativo comercial (labtest), e as leituras das amostras foram realizadas em espectofotômetro (E-225-D, CELM).

As análises hemogasométricas foram realizadas em todas as amostras logo após coleta, e com 1, 2, 3, 4, 6, 8, 10, 12, e 24 horas, utilizando-se para tanto o analisador de $\mathrm{pH}$ e gases sangüíneos ${ }^{10}$, o qual é capaz de mensurar o $\mathrm{pH}$ e as pressões parciais de oxigênio $\left(\mathrm{PO}_{2}\right)$ e dióxido de carbono $\left(\mathrm{PCO}_{2}\right)$ através de um sistema de eletrodos (ABL 5, Radiometer, Copenhagen) e de calcular, automaticamente $^{5}$, os valores das demais variáveis: total de dióxido de carbono no plasma $\left(\mathrm{TCO}_{2}\right)$, concentração de bicarbonato plasmático $\left(\mathrm{HCO}_{3}{ }^{-}\right)$, excesso ou déficit de bases no sangue (BE), concentração de bicarbonato padrão no sangue (StB) e saturação de oxigênio no sangue ( $\mathrm{Sat} \mathrm{O}_{2}$ ).

Em seguida a introdução da alíquota 
Tabela 1 - Valores médios, desvios-padrão e contrastes entre médias do $\mathrm{pH}$, pressões parciais de dióxido de carbono $\left(\mathrm{PCO}_{2}\right)$ e de oxigênio $\left(\mathrm{PO}_{2}\right)$ bicarbonato $\left(\mathrm{HCO}_{3}\right)$, total de dióxido de carbono $\left(\mathrm{TCO}_{2}\right)$, excesso ou déficit de base (BE), bicarbonato padrão (StB) e saturação de oxigênio $\left(\mathrm{SatO}_{2}\right)$ nas amostras de sangue de ovinos mantidas a temperatura ambiente $\left(\mathrm{T} 1-23 \mathrm{a} 25^{\circ} \mathrm{C}\right)$ ou sob refrigeração (T2 - 0 a $3^{\circ} \mathrm{C}$ ) ao longo do período de 24 horas. São Paulo, 2004

\begin{tabular}{|c|c|c|c|c|c|c|c|c|c|c|c|c|}
\hline \multirow{2}{*}{\multicolumn{2}{|c|}{ Variáveis }} & \multicolumn{11}{|c|}{ Tempo (horas) } \\
\hline & & 0 & 1 & 2 & 3 & 4 & 5 & 6 & 8 & 10 & 12 & 24 \\
\hline \multirow[t]{2}{*}{$\mathrm{pH}$} & $T_{1}$ & $7,35 \pm 0,013^{a}$ & $7,34 \pm 0,013^{\text {abc }}$ & $7,33 \pm 0,013$ alc & $7,33 \pm 0,013$ abc & $7,31 \pm 0,012^{\mathrm{bcd}}$ & $7,30 \pm 0,012$ bcd & $7,30 \pm 0,012$ de & $7,28 \pm 0,012 \mathrm{def}$ & $7,27 \pm 0,012^{\text {ef }}$ & $5 \pm 0,013^{i}$ & $0,014^{8}$ \\
\hline & $T_{2}$ & $7,36 \pm 0,016^{2}$ & $7,36 \pm 0,016^{2}$ & $7,36 \pm 0,016^{2}$ & $7,36 \pm 0,015^{2}$ & $7,36 \pm 0,015^{2}$ & $7,35 \pm 0,015^{a}$ & $7,35 \pm 0,015^{2}$ & $7,35 \pm 0,016^{2}$ & $7,35 \pm 0,015^{\mathrm{a}}$ & $7,34 \pm 0,015^{a}$ & $7,34 \pm 0,015^{2}$ \\
\hline $\mathrm{PCO}_{2}$ & $T_{1}$ & $44,75 \pm 1,25^{8}$ & $45,41 \pm 1,32^{\text {is }}$ & $46,33 \pm 1,35^{\text {eff }}$ & $47,33 \pm 1,46^{\text {efs }}$ & $48,66 \pm 1,44^{\text {efs }}$ & $49,67 \pm 1,48^{\mathrm{efg}}$ & $50,25 \pm 1,44^{\mathrm{ced}}$ & $53,00 \pm 1,56^{\mathrm{kcd}}$ & $54,67 \pm 1,61^{\mathrm{bc}}$ & $56,42 \pm 1,66^{b}$ & $63,58 \pm 1,96^{a}$ \\
\hline $\mathrm{mmHg}$ & $T_{2}$ & $46,33 \pm 1,81^{\text {a }}$ & $46,17 \pm 1,80^{\mathrm{a}}$ & $46,17 \pm 1,80^{\mathrm{a}}$ & $46,75 \pm 1,83^{\text {a }}$ & $46,83 \pm 1,77^{a}$ & $46,58 \pm 1,76^{a}$ & $46,92 \pm 1,80^{\mathrm{a}}$ & $47,75 \pm 1,84^{\text {a }}$ & $47,92 \pm 1,84^{\mathrm{a}}$ & $48,17 \pm 1,87^{a}$ & $47,83 \pm 1,88^{\mathrm{a}}$ \\
\hline $\mathrm{PO}_{2}$ & $T_{1}$ & $44,58 \pm 4,57^{a}$ & $45,42 \pm 4,57^{a}$ & $44,91 \pm 4,27^{a}$ & $45,33 \pm 4,42^{\mathrm{a}}$ & $45,58 \pm 4,22^{\circ}$ & $45,58 \pm 4,05^{a}$ & $45,67 \pm 3,95^{a}$ & $46,25 \pm 3,81^{\mathrm{a}}$ & $46,67 \pm 3,69^{a}$ & $46,67 \pm 3,80^{a}$ & $46,83 \pm 3,16^{\mathrm{a}}$ \\
\hline $\mathrm{mnHg}_{\mathrm{m}}$ & $T_{2}$ & $38,83 \pm 1,03^{2}$ & $39,08 \pm 1,07^{2}$ & $39,33 \pm 1,06^{2}$ & $39,67 \pm 1,00^{2}$ & $39,58 \pm 1,07^{\mathrm{a}}$ & $39,83 \pm 1,08^{2}$ & $40,08 \pm 1,08^{2}$ & $40,08 \pm 1,08^{2}$ & $40,92 \pm 1,13^{a}$ & $40,58 \pm 1,11^{2}$ & $41,33 \pm 1,14^{2}$ \\
\hline $\mathrm{HCO}_{3}$ & $T_{1}$ & $23,58 \pm 0,50^{a}$ & $23,41 \pm 0,48^{a}$ & $23,41 \pm 0,54^{a}$ & $23,50 \pm 0,50^{a}$ & $23,58 \pm 0,50^{a}$ & $23,41 \pm 0,51^{\mathrm{a}}$ & $23,25 \pm 0,54^{\mathrm{a}}$ & $23,58 \pm 0,50^{\mathrm{a}}$ & $23,42 \pm 0,48^{\mathrm{a}}$ & $23,33 \pm 0,48^{a}$ & $22,58 \pm 0,50^{a}$ \\
\hline mmoli & $T_{2}$ & $24,92 \pm 0,43^{\text {a }}$ & $24,92 \pm 0,43^{3}$ & $24,83 \pm 0,44^{\mathrm{a}}$ & $24,75 \pm 0,42^{\mathrm{a}}$ & $24,83 \pm 0,44^{\mathrm{a}}$ & $24,75 \pm 0,39^{\mathrm{a}}$ & $24,67 \pm 0,37^{\mathrm{a}}$ & $24,83 \pm 0,44^{\text {a }}$ & $24,83 \pm 0,44^{a}$ & $24,83 \pm 0,44^{\mathrm{a}}$ & $24,25 \pm 0,43^{2}$ \\
\hline $\mathrm{TCO}_{2}$ & $T_{1}$ & $55,67 \pm 1,17^{\circ}$ & $55,10 \pm 1,18^{a}$ & $55,10 \pm 1,18^{a}$ & $55,25 \pm 1,18^{\circ}$ & $55,75 \pm 1,22^{\circ}$ & $55,50 \pm 1,13^{a}$ & $55,10 \pm 1,18^{a}$ & $55,92 \pm 1,21^{\circ}$ & $55,92 \pm 1,16^{a}$ & $55,92 \pm 1,13^{a}$ & $54,91 \pm 1,13^{\mathrm{a}}$ \\
\hline mmoll & $T_{2}$ & $58,83 \pm 0,94^{\mathrm{a}}$ & $58,50 \pm 1,00^{a}$ & $58,25 \pm 0,97^{a}$ & $58,67 \pm 0,99^{\mathrm{a}}$ & $58,75 \pm 1,02^{\text {a }}$ & $58,00 \pm 1,02^{\mathrm{a}}$ & $58,17 \pm 0,98^{\mathrm{a}}$ & $59,00 \pm 0,97^{\mathrm{a}}$ & $58,75 \pm 0,97^{\mathrm{a}}$ & $58,83 \pm 0,98^{\mathrm{a}}$ & $57,33 \pm 0,99^{\mathrm{a}}$ \\
\hline BE & $T_{1}$ & $-1,00 \pm 0,56^{a}$ & $-1,42 \pm 0,54^{\mathrm{ab}}$ & $-1,42 \pm 0,54^{\mathrm{ab}}$ & $-1,92 \pm 0,57^{\mathrm{abc}}$ & $-1,92 \pm 0,61^{a b c}$ & $-2,17 \pm 0,60$ abc & $-2,42 \pm 0,54^{\mathrm{abc}}$ & $-2,92 \pm 0,61 \mathrm{bc}$ & $-3,17 \pm 0,58 \mathrm{bc}$ & $-3,67 \pm 0,53^{c}$ & $-5,42 \pm 0,56^{d}$ \\
\hline mmoll & $T_{2}$ & $0,17 \pm 0,56^{\mathrm{a}}$ & $0,00 \pm 0,55^{\mathrm{a}}$ & $0,08 \pm 0,53^{\text {a }}$ & $0,00 \pm 0,52^{a}$ & $0,08 \pm 0,53^{\mathrm{a}}$ & $0,00 \pm 0,55^{\mathrm{a}}$ & $-0,17 \pm 0,53^{\text {a }}$ & $0,17 \pm 0,52^{\text {a }}$ & $-0,08 \pm 0,53^{\text {a }}$ & $-0,08 \pm 0,51^{\text {a }}$ & $-0,75 \pm 0,48^{2}$ \\
\hline StB & $T_{1}$ & $22,92 \pm 0,48^{\circ}$ & $22,67 \pm 0,53^{\text {ab }}$ & $22,58 \pm 0,53^{\mathrm{ab}}$ & $22,25 \pm 0,50^{a b c}$ & $22,25 \pm 0,50^{\mathrm{abc}}$ & $22,00 \pm 0,50^{\text {abc }}$ & $21,92 \pm 0,48$ atc & $21,58 \pm 0,53$ abc & $21,17 \pm 0,44^{\mathrm{bc}}$ & $20,83 \pm 0,47^{c}$ & $19,42 \pm 0,47^{\mathrm{d}}$ \\
\hline mmoli & $T_{2}$ & $23,83 \pm 0,49^{\mathrm{a}}$ & $23,83 \pm 0,47^{a}$ & $23,67 \pm 0,47^{a}$ & $23,75 \pm 0,49^{\mathrm{a}}$ & $23,83 \pm 0,47^{\mathrm{a}}$ & $23,75 \pm 0,42^{\mathrm{a}}$ & $23,67 \pm 0,47^{\mathrm{a}}$ & $23,83 \pm 0,47^{\mathrm{a}}$ & $23,75 \pm 0,48^{\mathrm{a}}$ & $23,66 \pm 0,47^{\mathrm{a}}$ & $23,25 \pm 0,48^{\mathrm{a}}$ \\
\hline $\mathrm{SatO}_{2}$ & $T_{1}$ & $68,92 \pm 3,51^{\circ}$ & $69,50 \pm 3,44^{\mathrm{a}}$ & $68,50 \pm 3,55^{a}$ & $68,41 \pm 3,63^{\circ}$ & $68,33 \pm 3,60^{\circ}$ & $68,25 \pm 3,57^{a}$ & $68,00 \pm 3,53^{a}$ & $67,75 \pm 3,54^{\circ}$ & $67,50 \pm 3,59^{a}$ & $66,33 \pm 3,55^{a}$ & $63,75 \pm 3,54^{a}$ \\
\hline 8 & $T_{2}$ & $65,08 \pm 2,35^{\text {a }}$ & $65,58 \pm 2,29^{\mathrm{a}}$ & $65,67 \pm 2,34^{a}$ & $65,83 \pm 2,30^{\mathrm{a}}$ & $66,25 \pm 2,25$ a & $66,33 \pm 2,28^{a}$ & $66,33 \pm 2,29^{\mathrm{a}}$ & $66,33 \pm 2,29^{\mathrm{a}}$ & $67,08 \pm 2,20^{\text {a }}$ & $66,42 \pm 2,21^{\mathrm{a}}$ & $67,42 \pm 2,23^{\mathrm{a}}$ \\
\hline
\end{tabular}

Letras não coincidentes na mesma linha denotam diferença significativa $(p \leq 0,05)$

de sangue no hemogasômetro foram corrigidos os valores da hemoglobina e da temperatura retal que o animal correspondente exibia um dia antes da coleta, pois os valores padrão assumidos pelo aparelho são referentes à espécie humana.

Os dados foram analisados por meio do programa computacional Statistical Analyses System (SAS Institut Inc. 2000), sendo primeiramente verificado a normalidade dos resíduos pelo teste de kolmogorov-Smirnov. Utilizou-se o modelo de análise de variância, verificando-se os efeitos de acondicionamento das amostras de sangue. Os contrastes entre médias foram realizados pelo d.m.s do teste de Duncan, considerando-se $\mathrm{p}<0,05$ como significativo.

\section{Resultados e Discussão}

Os resultados obtidos, para as variáveis hemogasométricas, em amostras de sangue venoso de ovinos mantidas a temperatura ambiente ou sob refrigeração, ao longo do período de 24 horas, são apresentados na tabela 1 e nas figuras 1 e 2 através de valores médios e desvios padrão.

As diferenças nos valores inicias, dos parâmetros hemogasométricos, entre as amostras de sangue mantidas a temperatura ambiente e aquelas conservadas entre 0 e $4^{\circ}$, deve-se provavelmente, à manipulação das amostras e ao erro intrínseco no momento da colheita, conforme já havia sido documentado por Haskins ${ }^{11}$ não ultrapassando, esses valores, os limites de significância.

Como pode ser evidenciado na tabela 1 e nas fig. 1 e 2, as amostras de sangue mantidas a temperatura ambiente sofreram alterações significativas ao longo dos tempos de análise, caracterizando-se por diminuições contínuas, a partir da 4, 8 e 10 horas após colheita para os valores do $\mathrm{pH}, \mathrm{BE}$ e StB, respectivamente, e por aumento, a partir $\mathrm{da}$ 6 hora, para os valores da $\mathrm{PCO}_{2}$ sendo esses resultados semelhantes aos obtidos por outros pesquisadores em trabalhos realizados com bovinos $^{14,5,6,7}$, eqüinos ${ }^{7,8}$, canino $^{3}$ e em suínos $^{2}$. A maioria desses autores ainda observaram uma diminuição significativa nos valores da $\mathrm{PO}_{2}$, fato não identificado no presente trabalho.

A manutenção do sangue por 24 horas à temperatura ambiente resulta em alterações no exame hemogasométrico que são decorrentes da atividade metabólica do sangue. In vitro essa atividade inclui o metabolismo aeróbio, com consumo de $\mathrm{O}_{2}$ 


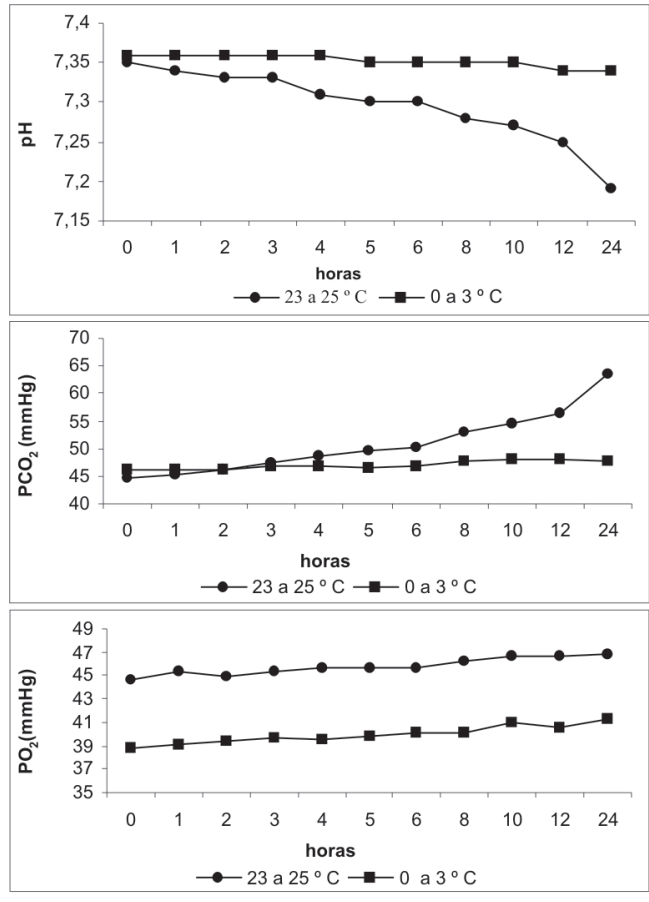

Figura 1 - Variações dos valores médios do $\mathrm{pH}$, pressões parciais de dióxido de carbono $\left(\mathrm{PCO}_{2}\right)$ e de oxigênio $\left(\mathrm{PO}_{2}\right)$, e bicarbonato $\left(\mathrm{HCO}_{3}\right)$ nas amostras de sangue venoso de ovinos mantidas a temperatura ambiente $\left(23 \mathrm{a} 25^{\circ} \mathrm{C}\right)$ ou sob refrigeração $\left(0 \mathrm{a} 3^{\circ} \mathrm{C}\right)$ ao longo do período de 24 horas

e produção de $\mathrm{CO}_{2}$ no ciclo dos ácidos tricarboxílicos e glicolise anaeróbia acompanhada da produção de metabólitos ácidos, principalmente os ácidos láctico e pirúrvico ${ }^{6}$. O acúmulo contínuo desses ácidos na corrente sangüínea pode, portanto, justificar as modificações observadas nos parâmetros hemogasométricos.

O consumo de oxigênio no sangue não refletiu significativamente nos valores da $\mathrm{PO}_{2}$. Possivelmente, esse fato ocorra devido a liberação do oxigênio da hemoglobina em conseqüência da diminuição do pH que diminui a afinidade da hemoglobina ao oxigênio (efeito de Bohr ${ }^{12}$ contrabalanceando a perda de oxigênio atribuída ao metabolismo aeróbio, resultando em discretas mudanças da $\mathrm{PO}_{2}$ já que o metabolismo do sangue de ovino, conforme observado nesta pesquisa, ocorre de forma menos intensa quando comparado com o de cão, eqüino, suíno e bovino, respectivamente.

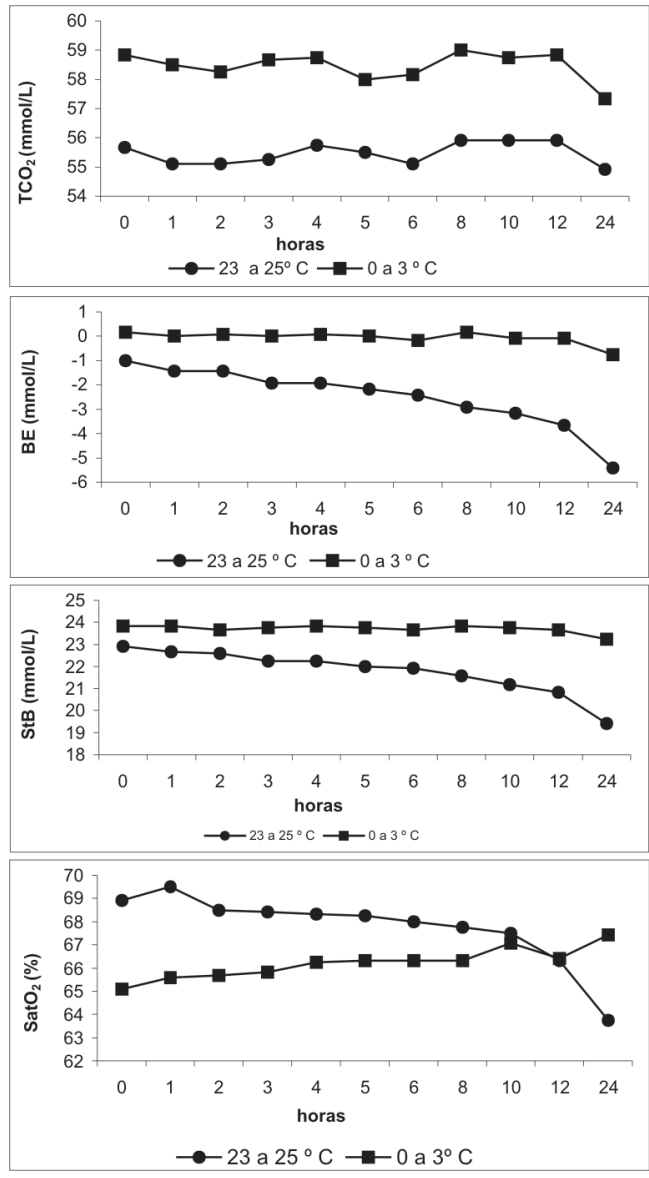

Figura 2 - Variação dos valores médios do total de dióxido de carbono $\left(\mathrm{TCO}_{2}\right)$, excesso ou déficit de base (BE), bicarbonato padrão (StB) e saturação de oxigênio $\left(\mathrm{SatO}_{2}\right)$ nas amostras de sangue venoso de ovinos mantidas a temperatura ambiente ( $23 \mathrm{a}$ $\left.25^{\circ} \mathrm{C}\right)$ ou sob refrigeração $\left(0\right.$ a $\left.3^{\circ} \mathrm{C}\right)$ ao longo do período de 24 horas

A manutenção do sangue venoso de ovino em água gelada reduziu drasticamente o metabolismo celular, de modo que não houve mudanças significativas nas variáveis heogasométricas no período de 24 horas de estudo. Esses resultados diferem dos obtidos por Kirnic et al. ${ }^{8}$ e Szenci et al. ${ }^{7,8}$ que obtiveram variação significativa a partir da sexta hora de conservação para os valores do $\mathrm{pH}$ e, mais tardiamente, para as demais veriáveis hemogasométricas. Essa discrepância nos resultados poderia ser atribuída a um provável reflexo das diferenças entre a metodologia utilizada em 
cada um dos experimentos.

No entanto, Szenci e Bresser ${ }^{6}$, trabalhando com sangue venoso de bovinos, concluíram que, do ponto de vista prático, o tempo de viabilidade das amostras conservadas poderia ser aceito por até 24 horas após colheita já que os valores médios de cada tempo não se distanciavam muito dos obtidos no momento de colheita. Desta maneira o exame hemogasométrico continuara a ter valor diagnóstico, contrariando o conceito de realizar a avaliação o mais rápido possível.

\section{Conclusões}

Os resultados obtidos neste estudo permite concluir que as amostras de sangue venoso de ovinos são viáveis, para a realização do exame hemogasométrico, até 24 horas após coleta, desde que mantidas sob adequada refrigeração.

\section{Effect of refrigeration on the hemogasometric examination of venous blood in sheep}

$\begin{array}{ll}\text { Abstract } & \text { Key-words: } \\ & \text { Ovine. } \\ & \text { Hemogasometry. } \\ \text { With the objective of evaluating the effect of refrigeration on the } & \text { Blood. } \\ \text { hemogasometric exam, venous blood samples were collected from } & \text { Refrigeration. }\end{array}$

12 healthy male sheep, Santa Ines breed, with a mean age of 4 months old, and body weight raging from 30 to $45 \mathrm{~kg}$. The blood samples for the hemogasometric examination were collected in two aliquots from each animal, using dispensable needles connected to plastic syringes containing about $1000 \mathrm{IU}$ sodium heparin. During and after the sampling, the care of avoiding the presence of air bubbles in the syringe was attempted. The samples without conservation were kept at room temperature, between 23 an $25^{\circ} \mathrm{C}$, and the samples under refrigeration were kept in an box containing $3 \mathrm{~L}$ of cold water and $3 \mathrm{Kg}$ of ice, to maintain a temperature between 0 and $4^{\circ} \mathrm{C}$. The hemogasometric analyses were made immediately after collection an after 1, 2, 3, 4, 5, 6, 8, 10, 12 and 24 hours. The results indicated significant alteration in samples kept at room temperature, characterized by decline, starting at 4, 8 and 10 hour post-collection, of the values $\mathrm{f} \mathrm{pH}, \mathrm{BE}$ and $\mathrm{StB}$, respectively, and by a raise, since the $6^{\text {th }}$ hour, of the values of $\mathrm{PCO}_{2}$. No significant variations of the parameters were seen in the refrigerated samples during the study. So, the conclusion is that ovine venous blood samples are viable, for the determination of the hemogasometric evaluation, until 24 hours after the collection, when kept (maintained) under adequate refrigeration.

\section{Referências}

1 LISBÔA, J. A. N. et al. Tempo de viabilidade de amostras de sangue venoso bovino destinadas ao exame hemogasométrico, quando mantidas em sob conservação em água gelada. Ciência Rural, v. 31, p. 271-276, 2001.

2 SUCUPIRA, M. C. A.; ORTOLANI, E. L. Uso do sangue arterial e venoso no exame do equilíbrio ácidobásico de novilhos normais ou com acidose metabólica. Ciência Rural, v. 33, n. 5, p. 863-868, 2003.

3 ASSAL, A. N.; CHRISTIANSEN, I. J.; POUSEN, J. S.
D. Acid-base status of porcine blood during storage. Nordisk Veterinaermedicin, v. 32, p. 9-16, 1980.

4 JAGOS, P.; BOUDA, J.; PRIKRYLOVA, J. The dynamics of the acid-base Changes of bovine venous blood in vitro, as depending on time. Veterinární Medicína, v. 22, p. 257-262, 1977.

5 POUSEND, J. S. D.; SURYNEK, J. Acid-base status of cattle blood. Sampling and storing. Nordisk Veterinaermedicin, v. 29, p. 271-2283, 1977.

6 SZENCI, O.; BESSER, T. Changes in blood gas and acid-base values of bovine venous blood during storage. Journal of the American Veterinary Medical 
Association, v. 197, p. 471-474, 1990.

7 SZENCI, O.; BRYDL, E.; BAJCSY, C. A. Effect of storage on measurement of ionized calcium and acidbase variabls in equine, bovine, ovine, and canine venous blood. Journal of the American Veterinary Medical Association, v. 199, p. 1167-1169, 1991.

8 KIRNIC, J. et al. Changes in the values of the characteristics of the acid-base status of sheep in the blood during storage at different temperatures. Veterinaria (Sarajevo), v. 30, n. 2, p. 237-246

9 ASSAL, A. N.; POUSEN, J. S. D. Acid-base status of equine blood during storage. Nordisk Veterinaermedicin, v. 30, p. 354-363, 1978

10 ASSAL, A. N.; ARNBJERG, J.; POUSEN, J. S. D. Acid-base status of canine blood during storage. Nordisk Veterinaermedicin, v. 30, p. 345-353, 1978.

11 HASKINS, S. C. Sampling and storage of blood for $\mathrm{pH}$ and blood gas analysis. Journal of the American Veterinary Medical Association, v. 170, p. 429-433, 1977.

12 KANEKO, J. J.; HARHEY, I. W.; BRUSS, M. L. Clinical biochemistry of domestic animal. 5 ed. San Diego: Academic Press, 1997. 932 p.

13 COLES, E. H. Patologia clínica veterinária. 3 ed. São Paulo: Manole, 1984. 566 p.

14 SEVERINGHAUS, J.W., BRADLEY, A.F. Electrodes for $\mathrm{pO}_{2}$ and $\mathrm{pCO}_{2}$ determination. Annales of Applyed Physiology, v. 13, p. 515-520, 1958.

15 SIGGAARD-ANDERSEN, O. Blood acid-base alignment nomogran. Scales for $\mathrm{pH}, \mathrm{pCO}_{2}$, base excess of whole blood of different hemoglobin concentrations, plasma bicarbonate, and plasma $\mathrm{CO}_{2}$. Scandinavian Journal of Clinical Laboratory Investigation, v. 15, p. 211-220, 1963. 